\title{
Network Analysis in Relationship Dynamics and Digital Social Identity in Youths
}

\author{
Rajendra Man Banepali' ${ }^{1}$ Subarna Shakya ${ }^{2}$, Gajendra Sharma ${ }^{3}$ \\ ${ }^{1}$ School of Computer Science and IT, Singhania University, Jhunjhunu, Rajasthan, India \\ ${ }^{2}$ Department of Electronics and Computer Engineering, Pulchowk Campus, Institute of Engineering \\ Tribhuvan University, Nepal \\ ${ }^{3}$ Department of Computer Science and Engineering, Kathmandu University, Nepal \\ Corresponding author: rajendra.banepali@yahoo.com,drss@ioe.edu.np,gajendra.sharma@ku.edu.np
}

Received: Nov 15, 2017 Revised: Dec 10, $2017 \quad$ Accepted: Dec 20, 2017

\begin{abstract}
People living with digital technology or without technology, no one is immune from digital technology nowadays. Netizen culture became a style of life in youth, the style as new behaviors. Aiming to scrutinize behavior of individual actor of digital network, this study has explored relationship dynamics of digital network observing students in survey. Network theory and cognition theory along with models from various sources have been employed to draw a result from data. Digital social identity and individual network behaviors like pattern of establishing new relation, altering relationship dynamics and termination are result of this work. In future, this model of network analysis can be expanded to various domains like political, intelligence and international relations.
\end{abstract}

Key words: Digital identity, network analysis, Netizen, relationship dynamics, social relationship

\section{Introduction}

Association, verbal or written based on interest, value or established social norms, between two people is relationship. Establishing new relationship, altering, and restricting relationship are activities of person in his relationship. Everyone is depended to another one for some purposes and we interact each other for a purpose using available media. Unlikely today, ancient time was hard for communication point of view. We are empowered by science and technology today and science fiction of few decades ago is a reality today using Information and Communication Technology (ICT) that we have.

We have though a lot of media nowadays, digital media became a major platform of information to the world about unfolding activities of people and nation. Social media for government and stateactors has opened a direct way to be connected with people in new way of entering social dialogue. The relation is expanding and altering for new beginning. Narrow downing ICT in this study, nexus between digital technology and social relationship has got attraction nowadays in research scholar. Here, I am taking an account of social relationship in a context of digital supremacy. Firstly, people relationship and extension of relationship with state-actors are major folds of this study in 
which network method is used to analyze relationship dynamics and its consequences. The major research question is how digital supremacy altering social relationship in real world.

\section{Literature Review}

This section has encompassed two thematic groups - theories and data from thesis for insight to draw a result. Putting cultural settings relating theories and data made concept to analyzed data.

\subsection{People in Netizen}

Modern life has been fastened everyone, especially youth, with new media along with its consequences. Youth has adopted new media, social network, in their life to translate their life into new way of making new connections, altering relations and restricting relations in digital world. Within decade of history of social media, social media migration has indicated that new upcoming generation has gained their unique experiences working with special content like text, audio and video media with new life style. With their unique identities in different platform for their path of career and life style, they have developed their own way to express emotions and concept of selfhood which is making new way of relationship dynamics nowadays. Their unique identity text platform like in messenger is making them efficient to learn and communicate and making vulnerable in cyberspace. Blog and website became a major platform to the world about unfolding activities of people nowadays. In a way, not only youth but also adults are also connected with many connections which shows importance to scrutinize relationship dynamics to maximize productivity of life reducing and preventive negative effects within networks since no one is immune from digital influences nowadays [7].

\subsection{Digital Supremacy}

Power is an influencing ability to let someone make decision. Does digital technology have power in a way? Observation is required to answer this question in scientific way what I tried to portray in this work. Wilson, 2008, stated that influencing power making life threat is hard-power life gun and military. Soft-power influences life indirectly for long term which translate life in new way. That is like money, economics and educations. New debate is going on about smart-power which is supposed to balance hard-power and soft-power in real life differently. World class researcher are wrestling to define smart power and how it is rendering the world activities preparing for new relationship dynamics [8].

\subsection{Social Relations}

Social relationship dynamics can be clearly observed in family which is smallest unit of social relationship but complex to analyze. It is not easy to analyze perfectly using available statistic tools and descriptive method adds clarity on analysis of individual network in family [7]. Here, I have included social relationship actualized and rendered by digital technology. That's why beyond family relationship, friends' circle relationship, observing individual networks, has been included into this work with network analysis [7]. 


\subsection{Network Theory and Implications}
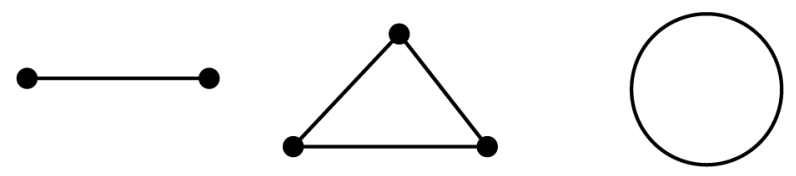

Fig. 1: Euler's Planar Network

Connection between vertices is network. Union set of finite set of coplanar paths is planar network consisting vertices, paths and regions. Vertices denotes individual nodes like person in society, path provides a meaning of link with another node (person) and region constitutes a context or an environment of the relationship like in Fig. $1[8,4]$.

Planar network formula provides tool to evaluate degree of strength and operation of relationship dynamics of network in which interaction of nodes are major to scrutinize in specific context. What's whey, we have network formal as statistical tool [2]. The following is the Euler's formula:

$V+R-P=2(V=$ Number of Vertices, $R=$ Number of Regions and $P=$ Number of Paths)

Network theory could be employed in both research paradigms - qualitative or quantitative - in which observation of individual network plays significant role while working for network analysis. The analysis does not deal attributes of nodes, the attributes like value of body, color and weight, are important in networks. It is limitation of network analysis. However, this tool has strength to explore network operations like cooperation, hostile, predatory, competitive, aggressive, interaction frequencies, magnitude of strength and conflict of network [6]. Studying individual network is important while analyzing effectiveness of network, interaction and outbreak of information into the network. A node having huge network can outbreak information fast covering huge geolocation in network [6].

\subsection{Meaning Making in Social Network}

\subsubsection{Cognition}

"Cognitive Psychology is the study of thinking and the processes underlying mental events." - Free Software Foundation (2008). Is it possible to make any meaning without anything? The question buzzed our mind for a second. We make meaning though sensitizing using our five sensitizing organs through mental process. Making meaning is a learning like reading book which is process of text and base on the text we make picture in our mind. The learning can be short term and long term to get solution for a problem [5]. There might be lot strategies to find out answers - right or wrong and sometimes the problem can also have distinct answer rather than right or wrong. Such answer could be found breaking the context [6] and analyze them rearranging for answer. This novel process, de-constructivism, provides new answer [7].

\subsubsection{Constructivism}

Learning process involves context or settings or environment. That is known as knowledge. We make meaning according to context which is based on time and place - called environment. The knowledge depends upon individual experiences and environment he is. That's why knowledge always has multiple realities which might be fit for me and might not be fit for other or meaning of same thing might be different to individual. Constructivism brings multiple realities of everything 
in different scenarios [1]. In a brief, this work tried to draw multiple realities in youth's behavior created by digital technology.

\subsection{Data}

These data in this paper are taken from [1] for conflict studies. Number of responders are 15 in semi-structural and open-ended questionnaires during survey and group discussion in which majority of students are 22 years and they are almost in same age group from 21 to 24 years [1].

\subsubsection{Digital Supremacy}

Researcher has tried to figure out in numeric values of uses and spending maximum time of digital technology implementing eleven questions in survey and in interview with individual. Researcher found influences of digital technology has been strongly skewed toward digital supremacy positively by value of 52.5 percent [1].

\subsubsection{Relationship Dynamics}

Researcher has limited social relations and conversation within family and friends in this tenure. According to survey, digital mediation - which is significantly visible by a value of 50.67 per cent in students - in conversation of family and friends is major observation of this section [5]. Observation of digital influence in family social functions, conversation, conflict within family, over sighting own activities, and consequences. Also, sometime negation feeling of digital impression within students was visible during observation of consequences of digital technology in their daily life and long-term timing with the technology.

\subsubsection{Mediation of Digital Technology}

Researcher has partially explored multiple realities - as any consequences especially in youth - of digital technology. Most of the answer revealed very interesting facts of youth such as connection with friends and peers, modeling of relations with friends, and exchange of feelings. According to survey, Influences of digital technology in social relations, especially within youth, is more approximately 58.33 per cent social relations like within friends and peers [1].

\subsubsection{Distribution of Actors}

Along with new structural change in communication channel, emerging digital technology has burst new digital social actor in digital world. Upcoming "Digital Social Identity" (DSI) is creating new actors in social interaction. Digital technology is demanding share of new actors in the society with their new Social Digital Identity, for example; according to result, digital technology has challenged the underlying assumptions and distribution of actors in social relations - and its result table, 64.45 percent share of new distribution of actors in many ways - Facebook profile, Blog profile and posting, LinkedIn profile, and other writer for other web - and maintain their identity as well [1].

\subsubsection{Null Digital State}

It is not imagination of without digital technology or people without digital technology. Researcher tried to explore absence of digital technology in the world of digital technology and people living with digital technology, it means why people living with digital technology ignore or leave the technology. Here by, the world without digital technology is coined as Null Digital Stage (NDW). 
According to survey, students, who are living without digital interruption in different contexts, are 37.78 percent [1].

\section{Methodology}

This study has encompassed two approaches - model building with short spam of textual data and numeric data. Theories are included to analyze data in a framework of approached theories like network theory and constructivism. Network theory is in center to analyze students' behaviors in their network working in social media which affect their real life too. Cognition theory and constructivism was coined to visualize how students make meaning of an object or event in their relationships and how they act. Data and model from [1] have been included to scrutinize for a result. Those data have been seriously analyzed employing network theory and cognition theory.

\section{Result}

\subsection{Digital Social Identity}

Societal reality got a new shaped with digital technology. New vision and boundless identity Digital Social Identity - because new prominent actor which is playing significant role into our life to reflect hyper reality. Hyper reality is reflection of real life in digital space, as node in network. Digital social identity is the entry point for digital network for individual, group and family. The identity is strong casting of societal reality and it also reflect to real life. It's like a loop. Digital social identity has not only reflection of attribute of societal reality but also shaped with new identity and behavior like it may not have political boundary or limited to any identification within limited geography. It does not have shape and limitation to act in network. Observing all presentations and study [1], digital social identity and its reflection are playing a vital role in network behavior since the reflection seems knowledge making from the context which effects behavior of network of individual. That's why it is very important to analyze action of network behavior.

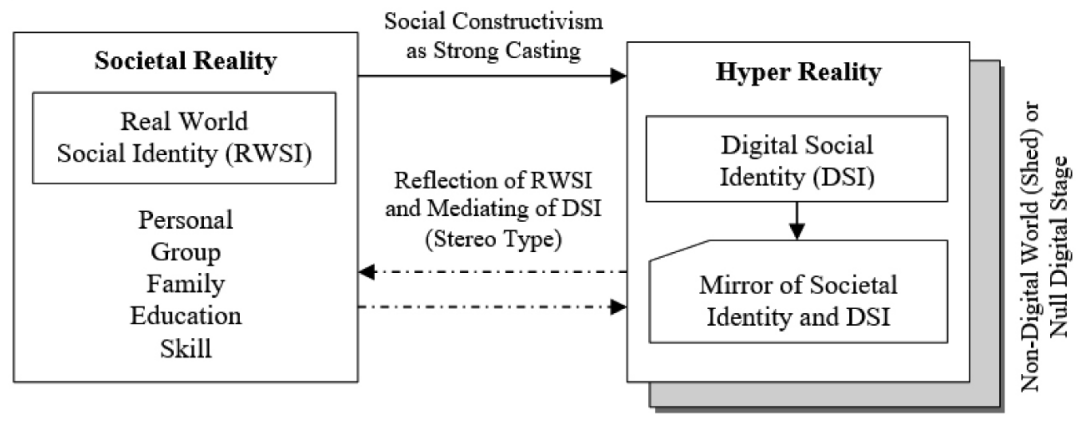

Fig. 2: Digital Social Identity Construction and Hyper Reality [1]

\subsection{Network Behavior}

Though attributes of node (actor or individual) are important to analyze, network analysis does not focus on those things and focus on action of actors which mediates network relationship and cooperation. To analyze whole network behavior, we should able to analyze individual network and his actions within overall core framework of network theory. What's why, I am portrayed result here in a similar manner. I have identified three network actions as individual behaviors which alter relationship in network. I have taken those actions in digital networks which alter relationship in real world so that I could measure digital influences in this work. 


\subsubsection{New Relationship}

Youth like engineering students in survey are in digital media with their digital social identity according to survey data. They are using different social media for establishing new known relationship and unknown digital space. Majority of students spend significant time in social networking which is approximately nearby college time. Observing student's data in research question \#1, about digital supremacy, it says $52.2 \%$ occupancy of digital influences in real life. Individual is very keen to use not only one but also many social media platforms to establish relationship in network. Temptation of their attachment with social media is her according to their statements and have gone deeper to translate their life into digital platform.

Observing individual social affairs and the connection of digital technology, the technology is contributing $50.67 \%$ in social calendars. And individual is also interesting with new platform for different purposes like they use Facebook for friends \& family, twitter for information and Instagram for closed-friends \& group to share emotions. Among those students $64.45 \%$ students seek for new digital identity to relate to people. They are expanding their network with emerging tool and new identity which has no boundary in terms of governance too.

\subsubsection{Altering Relationship}

In significant digital supremacy, likely $52.2 \%$, students found many ways and causes to reaffirm their relationship in social network with people. Getting rest from social media and de-activating digital identity is higher in youth. More than $58.33 \%$ student have faced consequences of digital mediation in their social relationship. Some students were very eager to spending time in social media and some were reluctant to spend significant time in social media according to your statement. However, they are though in least time in digital media, they real life has also effects of digital identities nowadays.

\subsubsection{Terminating Relationship}

In this section, I have tried to draw how digital platform became a tool to influence students to affirm the status of relationship to the end. It has also effects in real life not only in digital platform. In a connection of causes of terminations of relationship, there might be many causes but in a lens of technology, the rapid digital mediation in social life is also a cause, for example; a student lost a friend. I came to know that few students stop using digital media interfering their life, because they want to get peace with no body. They disconnected temporary with everyone. The termination might be hard or soft. Hard termination could affect even in real social life and soft termination is like a break which was observed in data.

More than $37.78 \%$ students avoid being out digital technology for their different requirements of life. Some wants peace without the technology and some needs a break for study. The state without the digital technology is Null Digital State in which privacy, anxiety, productivity and family intimacy are major ingredients. Privacy plays an important role working in digital platform to determinate or altering relationship. Most of the people do not initiate their digital journey because of privacy. Many people take an account of privacy in digital platform very seriously before their step in. And someone disconnects in network too. Some students do not want to connect to avoid anxiety from unknown connections. Anxiety is major to enforce people to reaffirm the status of relationship.

Since new media like social media has occupied significant time of students, they were hurt in 
exam. Productivity was a question to them. A student determinate all social media network just to focus on study but not relationship of real life. A student has also advised to control the digital media for their productivity of life ensuring security of data - privacy.

\section{Conclusion and Future Work}

Increasing digital culture came with new power - like digital supremacy - which is rendering our life in new direction and behavior. Set of new culture is significantly visible in youth nowadays and they have developed their own style to approach and express their emotions in their network since learning from network is also very different with their digital social identity which is socially constructed in digital world reflecting societal realities. In this work, data are acquired from [1], to draw a result in a lens of network theory. As a result, reflection of digital social identity is in front as eye opening that how the identity is constructed and its reflection as well as individual behaviors, like establishing, altering and terminating relationship, in network are major issues.

This study has worked with limited networks and one region to simplify how digital social network works in students. However, it could be expanded in many domains like political, national security, international relations and intelligence to visualize network behaviors so that we could prevent future conflict for better world.

\section{References}

[1] Banepali RM (2016), Digital Social Identity: Conversation Model of Social Relations Among Youth in Kathmandu Shaped by Digital Technology, Department of Conflict, Peace and Development Studies, Master Thesis, Tribhuvan University, Nepal

[2] Charles AR (1974), Network Theory - An Enrichment Topic, National Council of Teachers of Mathematics, 67(2): 175-178

[3] Davis, Weigel K, James M and Gardner CH (N/A), Social Development in the Era of New Digital Media

[4] Free Software Foundation (2008), Cognitive Psychology and Cognitive Neuroscience, USA: Free Software Foundation, Inc.

[5] Keith ST (2011), Constructivism as Educational Theory: Contingency in Learning, and Optimally Guided Instruction, New York: Nova Science Publishers

[6] Krause J, Croft DP and James R (2007), Social Network Theory in the Behavioural Science: Potential Applications, Behavioral Ecology and Sociobiology, 62(1): 15-27

[7] Snijders TAB and Kenny DA (1999), The Social Relations Model for Family Data: A Multiple Approach, University of Groningen and University of Connecticut, 6: 471-486

[8] Wilson EJ (2008), Hard Power, Soft Power and Smart Power, Annals of the American Academy of Political and Social Science, 616: 110-124 